DAMTP/94-105, hep-th/9412167, and Phys.Rev.D52:1108 (1995)

\title{
Hilbert Space Representation of the Minimal Length Uncertainty Relation
}

\author{
Achim Kempet, Gianpiero Mangano, Robert B. Mann目 \\ Department of Applied Mathematics \& Theoretical Physics \\ University of Cambridge \\ Cambridge CB3 9EW, U.K.
}

\begin{abstract}
The existence of a minimal observable length has long been suggested in quantum gravity as well as in string theory. In this context a generalized uncertainty relation has been derived which quantum theoretically describes the minimal length as a minimal uncertainty in position measurements. Here we study in full detail the quantum mechanical structure which underlies this uncertainty relation.
\end{abstract}

*supported by Studienstiftung des Deutschen Volkes, BASF-fellow, a.kempf@amtp.cam.ac.uk

${ }^{\dagger}$ supported by University of Naples, Federico II, Italy, g.mangano@amtp.cam.ac.uk

${ }^{\ddagger}$ supported by NSERC of Canada, rbm20@amtp.cam.ac.uk 


\section{Introduction}

One of the major problems in quantum gravity is that the introduction of gravity into quantum field theories appears to spoil their renormalizability. On the other hand it has long been suggested that gravity itself should lead to an effective cutoff in the ultraviolet, i.e. to a minimal observable length. The argument is based on the expectation that the high energies used in trying to resolve small distances will eventually significantly disturb the spacetime structure by their gravitational effects. While conventional spacetime locality seems to be probed down to scales of about 1 $\mathrm{TeV}$ [1] it is quite clear that this type of effect ought to occur at least at energy scales as large as the Planck scale. If indeed gravity induces a lower bound to the possible resolution of distances, gravity could in fact be expected to regularize quantum field theories rather than rendering them nonrenormalizable. It is a natural, though nontrivial assumption that a minimal length should quantum theoretically be described as a nonzero minimal uncertainty $\Delta x_{0}$ in position measurements. String-theoretic arguments also lead to a minimal length effectively of the form of a minimal position uncertainty. See e.g. [2]-[7] and for a recent survey [8].

The purpose of this paper is to develop a generalized quantum theoretical framework which implements the appearance of a nonzero minimal uncertainty in positions. We will here confine ourselves to exploring the implications of such a minimal uncertainty in the context of non-relativistic quantum mechanics. A further paper extending the framework to field theory is in preparation.

Our analysis is motivated by the results of [9]-[12] where the more general case, which includes nonzero minimal uncertainties in momenta as well as position, was considered. This general case is far more difficult to handle, since (as we will explain) neither a position nor a momentum space representation is viable. Instead one has to resort to a generalized Bargmann-Fock space representation. The construction of generalized Bargmann-Fock Hilbert spaces was realized by making use of algebraic techniques developed in the field of quantum groups; for a comparison see e.g. [13][17]. However, using the discrete Bargmann Fock basis, actual calculations typically involve finite difference equations and infinite sums rather than differential equations and integrals. For these technical reasons it has so far only in examples been possible to prove e.g. that ultraviolet divergences may be regulated using this approach. In the present paper we consider only the case of minimal uncertainty in position, taking the minimal uncertainty in momentum to vanish. This case is of interest because there still exists a continuous momentum space representation. Hence it allows us to explore the physical implications of a minimal length in a manner that is technically much easier to handle than the general case. 


\section{Minimal length uncertainty relations}

In one dimension the simplest generalized uncertainty relation which implies the appearance of a nonzero minimal uncertainty $\Delta x_{0}$ in position has the form:

$$
\Delta x \Delta p \geq \frac{\hbar}{2}\left(1+\beta(\Delta p)^{2}+\gamma\right)
$$

where $\beta$ and $\gamma$ are positive and independent of $\Delta x$ and $\Delta p$ (but may in general depend on the expectation values of $\mathbf{x}$ and $\mathbf{p}$ ). The curve of minimal uncertainty is illustrated in Fig. 1.

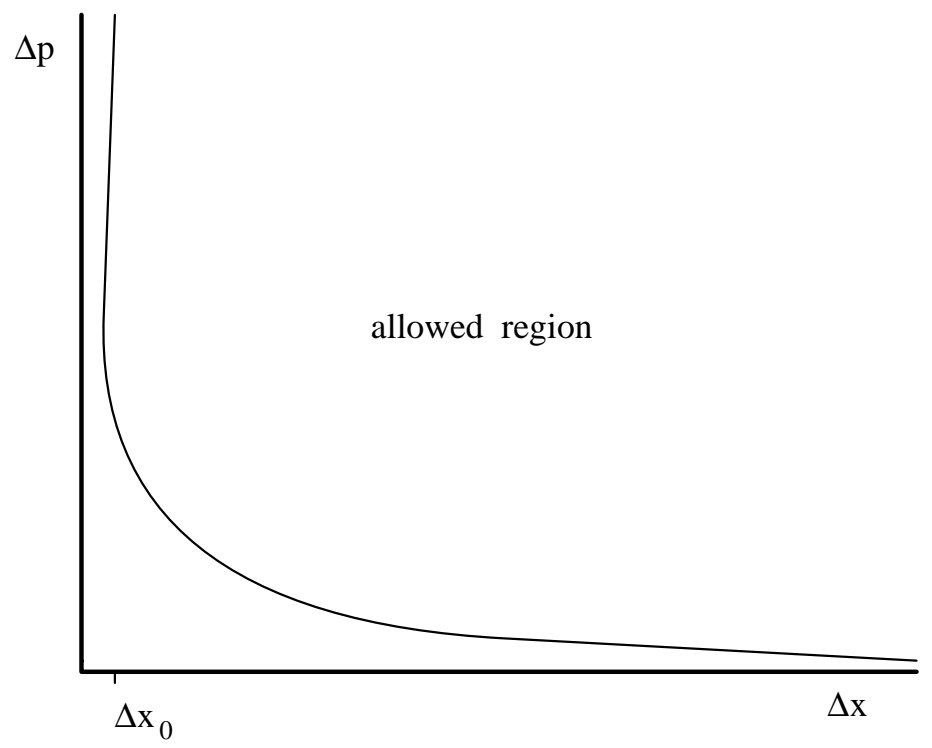

Fig1: Modified uncertainty relation, implying a 'minimal length' $\Delta x_{0}>0$

While in ordinary quantum mechanics $\Delta x$ can be made arbitrarily small by letting $\Delta p$ grow correspondingly, this is no longer the case if (11) holds. If for decreasing $\Delta x$ $\Delta p$ increases, the new term $\beta(\Delta p)^{2}$ on the rhs of (11) will eventually grow faster than the lhs. Hence $\Delta x$ can no longer be made arbitrarily small.

This type of generalized uncertainty relation has appeared in the context of quantum gravity and string theory, see e.g. [7] and independently from formal considerations in ref. [11]. It allows one to express the (non-trivial) idea that a minimal length should quantum theoretically be described as a minimal uncertainty in position measurements.

More generally, the relation

$$
\Delta x \Delta p \geq \frac{\hbar}{2}\left(1+\alpha(\Delta x)^{2}+\beta(\Delta p)^{2}+\gamma\right)
$$

leads to a nonzero minimal uncertainty in both position $\Delta x_{0}$ and momentum $\Delta p_{0}$ for $\alpha>0$. 
Now in general it is known that for any pair of observables $A, B$ which are represented as symmetric operators on a domain of $A^{2}$ and $B^{2}$ the uncertainty relation

$$
\Delta A \Delta B \geq \frac{\hbar}{2}|\langle[A, B]\rangle|
$$

will hold. In particular, we see that commutation relations of the form

$$
[\mathbf{x}, \mathbf{p}]=i \hbar\left(1+\alpha \mathbf{x}^{2}+\beta \mathbf{p}^{2}\right)
$$

underly the uncertainty relation (2) with $\gamma=\alpha\langle\mathbf{x}\rangle^{2}+\beta\langle\mathbf{p}\rangle^{2}$.

\section{$3 \quad$ Hilbert space representation}

We will now construct a Hilbert space representation of such a commutation relation. Let us clarify that we generally require physical states not only to be normalizable, but to also have well defined expectation values of position and momentum and also well defined uncertainties in these quantities. It is important to note that this implies that physical states always lie in the common domain $D_{\mathbf{x}, \mathbf{x}^{2}, \mathbf{p}, \mathbf{p}^{2}}$ of the symmetric operators $\mathbf{x}, \mathbf{p}, \mathbf{x}^{2}, \mathbf{p}^{2}$. On $D_{\mathbf{x}, \mathbf{x}^{2}, \mathbf{p}, \mathbf{p}^{2}}$ the uncertainty relation holds from which we can already conclude that physical states are constrained to the 'allowed' region of Fig. 1. Actually we can further derive from the uncertainty relation a severe contraint on the possible ansatzes for Hilbert space representations.

\subsection{Representation theoretic consequences of the uncer- tainty relations}

In ordinary quantum mechanics $\mathbf{x}$ and $\mathbf{p}$ could e.g. be represented as multiplication or differentiation operators acting on square integrable position- or momentum- space wave functions $\psi(x):=\langle x \mid \psi\rangle$ or $\psi(p):=\langle p \mid \psi\rangle$, where the $|x\rangle$ and $|p\rangle$ are position and momentum 'eigenstates'. Strictly speaking the $|x\rangle$ and $|p\rangle$ are not physical states since they are not normalizable and thus not in the Hilbert space. However, the operators $\mathbf{x}$ and $\mathbf{p}$ are essentially self-adjoint and the 'eigenstates' can be approximated to arbitrary precision by sequences $\left|\psi_{n}\right\rangle$ of physical states of increasing localization in position or momentum space:

$$
\lim _{n \rightarrow \infty} \Delta x_{\left|\psi_{n}\right\rangle}=0
$$

or

$$
\lim _{n \rightarrow \infty} \Delta p_{\left|\psi_{n}\right\rangle}=0
$$

As has been pointed out in [11, 12] this situation changes drastically with the introduction of minimal uncertainties $\Delta x_{0} \geq 0$ and/or $\Delta p_{0} \geq 0$. For example a nonzero minimal uncertainty in position

$$
(\Delta x)_{|\psi\rangle}^{2}=\left\langle\psi\left|(\mathbf{x}-\langle\psi|\mathbf{x}| \psi\rangle)^{2}\right| \psi\right\rangle \geq \Delta x_{0} \quad \forall|\psi\rangle
$$


implies that there cannot be any physical state which is a position eigenstate since an eigenstate would of course have zero uncertainty in position.

Of course this does not exclude the existence of unphysical, 'formal position eigen-

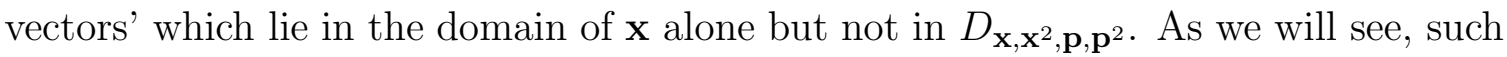
formal $\mathbf{x}$-eigenvectors do exist and are of infinite energy. Most importantly however, unlike in ordinary quantum mechanics, it is no longer possible to approximate formal eigenvectors through a sequence of physical states of uncertainty in positions decreasing to zero. This is because now all physical states have at least a finite minimal uncertainty in position.

Technically, as we will see, a minimal uncertainty in position will mean that the position operator is no longer essentially self-adjoint but only symmetric. While the preservation of the symmetry insures that all expectation values are real, giving up self-adjointness opens the way for the introduction of minimal uncertainties.

However, since there are then no more position eigenstates $|x\rangle$ in the representation of the Heisenberg algebra, the Heisenberg algebra will no longer find a Hilbert space representation on position wave functions $\langle x \mid \psi\rangle$. Similarly a minimal uncertainty in momentum also abandons momentum space wave functions. In this general case we therefore resorted to a generalized Bargmann Fock representation [11, 12.

Here we will restrict ourselves to the case $\alpha=0$ where there is no minimal uncertainty

in momentum. This will allows us to work with the convenient representation of the commutation relations on momentum space wave functions.

As we will see, the states of maximal localization will be proper physical states. We can use them to define a 'quasi-position' representation. This representation has a direct interpretation in terms of position measurements, although it does of course not diagonalise $\mathbf{x}$.

\subsection{Representation on momentum space}

We consider the associative Heisenberg algebra generated by $\mathbf{x}$ and $\mathbf{p}$ obeying the commutation relation $(\beta>0)$

$$
[\mathbf{x}, \mathbf{p}]=i \hbar\left(1+\beta \mathbf{p}^{2}\right)
$$

The corresponding uncertainty relation is

$$
\Delta x \Delta p \geq \frac{\hbar}{2}\left(1+\beta(\Delta p)^{2}+\beta\langle\mathbf{p}\rangle^{2}\right)
$$

with the curve on the boundary of the allowed region being (see Fig.1):

$$
\Delta p=\frac{\Delta x}{\hbar \beta} \pm \sqrt{\left(\frac{\Delta x}{\hbar \beta}\right)^{2}-\frac{1}{\beta}-\langle\mathbf{p}\rangle^{2}}
$$


One reads off the minimal position uncertainty

$$
\Delta x_{\min }(\langle\mathbf{p}\rangle)=\hbar \sqrt{\beta} \sqrt{1+\beta\langle\mathbf{p}\rangle^{2}}
$$

so that the absolutely smallest uncertainty in positions has the value

$$
\Delta x_{0}=\hbar \sqrt{\beta}
$$

There is no nonvanishing minimal uncertainty in momentum. In fact the Heisenberg algebra can be represented on momentum space wave functions $\psi(p):=\langle p \mid \psi\rangle$.

We let $\mathbf{p}$ and $\mathbf{x}$ act as operators

$$
\begin{aligned}
& \mathbf{p} \cdot \psi(p)=p \psi(p) \\
& \mathbf{x} \cdot \psi(p)=i \hbar\left(1+\beta p^{2}\right) \partial_{p} \psi(p)
\end{aligned}
$$

on the dense domain $S_{\infty}$ of functions decaying faster than any power. This representation is easily seen to respect the commutation relation (6). Note that, although the generalization for commutation relations of the type $[\mathbf{x}, \mathbf{p}]=i \hbar f(\mathbf{p})$ seems obvious, such generalizations are representation-theoretically nontrivial, in particular if $f$ is not strictly positive. This will be studied elsewhere.

Further, $\mathbf{x}$ and $\mathbf{p}$ are symmetric on the domain $S_{\infty}$

$$
(\langle\psi| \mathbf{p})|\phi\rangle=\langle\psi|(\mathbf{p}|\phi\rangle) \quad \text { and } \quad(\langle\psi| \mathbf{x})|\phi\rangle=\langle\psi|(\mathbf{x}|\phi\rangle)
$$

but now with respect to the scalar product:

$$
\langle\psi \mid \phi\rangle=\int_{-\infty}^{+\infty} \frac{d p}{1+\beta p^{2}} \psi^{*}(p) \phi(p)
$$

The symmetry of $\mathbf{p}$ is obvious. The symmetry of $\mathbf{x}$ can be seen by performing a partial integration.

$$
\int_{-\infty}^{+\infty} \frac{d p}{1+\beta p^{2}} \psi^{*}(p) i \hbar\left(1+\beta p^{2}\right) \partial_{p} \phi(p)=\int_{-\infty}^{+\infty} \frac{d p}{1+\beta p^{2}}\left(i \hbar\left(1+\beta p^{2}\right) \partial_{p} \psi(p)\right)^{*} \phi(p)
$$

Thereby the $\left(1+\beta p^{2}\right)^{-1}$-factor of the measure on momentum space is needed to cancel a corresponding factor of the operator representation of $\mathbf{x}$.

The identity operator can thus be expanded as

$$
1=\int_{-\infty}^{+\infty} \frac{d p}{1+\beta p^{2}}|p\rangle\langle p|
$$

and the scalar product of momentum eigenstates is therefore:

$$
\left\langle p \mid p^{\prime}\right\rangle=\left(1+\beta p^{2}\right) \delta\left(p-p^{\prime}\right)
$$

While the momentum operator still is essentially self-adjoint, the functional analysis of the position operator, as expected from the appearance of the minimal uncertainty in positions, changes. 


\subsection{Functional analysis of the position operator}

The eigenvalue problem for the position operator takes, on momentum space, the form of the differential equation:

$$
i \hbar\left(1+\beta p^{2}\right) \partial_{p} \psi_{\lambda}(p)=\lambda \psi_{\lambda}(p)
$$

It can be solved to obtain formal position eigenvectors:

$$
\psi_{\lambda}(p)=c e^{-i \frac{\lambda}{\hbar \sqrt{\beta}} \tan ^{-1}(\sqrt{\beta} p)}
$$

They are normalizable:

$$
1=c c^{*} \int_{-\infty}^{+\infty} \frac{1}{1+\beta p^{2}}=c c^{*} \pi / \sqrt{\beta}
$$

Thus

$$
\psi_{\lambda}(p)=\sqrt{\frac{\sqrt{\beta}}{\pi}} e^{-i \frac{\lambda}{\hbar \sqrt{\beta}} \tan ^{-1}(\sqrt{\beta} p)}
$$

We know however from the uncertainty relation that these formal eigenvectors are not physical states. Let us first proceed with a formal analysis.

We note that there is exactly one eigenvector to each of the eigenvalues $\pm i$ and that they are normalizable. Technically this means the following: The operator $\mathbf{x}^{* *}$ which is the bi-adjoint of the densely defined symmetric operator $\mathbf{x}$ is symmetric and closed and has non-empty deficency subspaces [11]:

$$
L_{ \pm i, \mathbf{x}^{* *}}^{\perp}:=\operatorname{ker}\left(\mathbf{x}^{*} \mp i\right) \cdot D_{\mathbf{x}^{*}}
$$

Here we used that $\mathbf{x}^{* * *}=\mathbf{x}^{*}$ which holds since $\mathbf{x}^{*}$ is closed and defined on a dense domain. From the dimensionalities of these subspaces, i.e. since the deficency indices are $(1,1)$, one concludes that the position operator is no longer essentially self-adjoint but has a one-parameter family of self-adjoint extensions instead.

This functional analytic structure had already been found and studied in detail in [11] where the more general case including also a minimal uncertainty in momentum has been covered.

It is however one of the advantages of our specialization to the case without a minimal uncertainty in momenta, that we can now construct the one-parameter family of diagonalisations of $\mathbf{x}$ explicitly. To this end we calculate the scalar product of the formal position eigenstates $\left|\psi_{\lambda}\right\rangle$ (see Fig.2)

$$
\begin{aligned}
\left\langle\psi_{\lambda^{\prime}} \mid \psi_{\lambda}\right\rangle & =\frac{\sqrt{\beta}}{\pi} \int_{-\infty}^{+\infty} \frac{d p}{1+\beta p^{2}} e^{-i \frac{\left(\lambda-\lambda^{\prime}\right)}{\hbar \sqrt{\beta}} \tan ^{-1}(\sqrt{\beta} p)} \\
& =\frac{2 \hbar \sqrt{\beta}}{\pi\left(\lambda-\lambda^{\prime}\right)} \sin \left(\frac{\lambda-\lambda^{\prime}}{2 \hbar \sqrt{\beta}} \pi\right)
\end{aligned}
$$




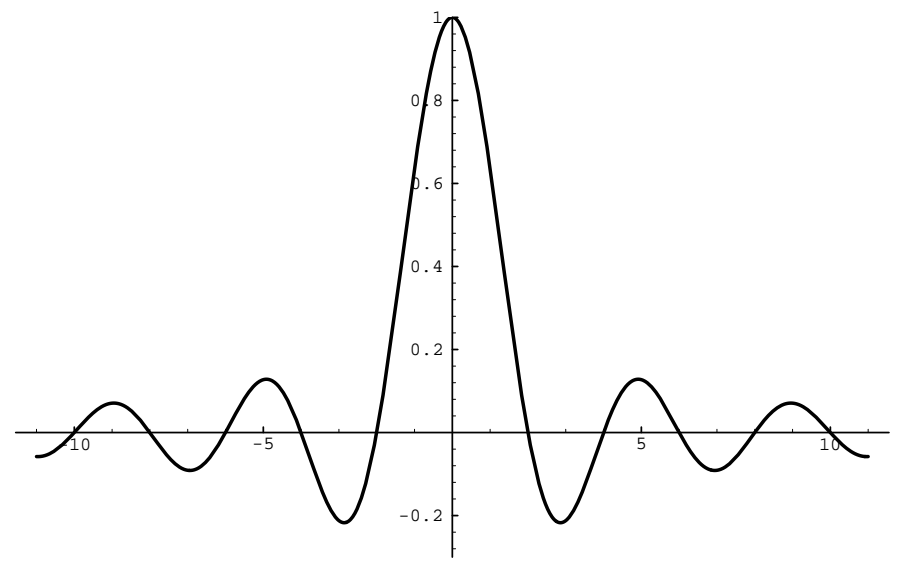

Fig2: Plotting $\left\langle\psi_{\lambda} \mid \psi_{\lambda^{\prime}}\right\rangle$ over $\lambda-\lambda^{\prime}$ in units of $\hbar \sqrt{\beta}=\Delta x_{0}$

Note that this curve is the special case $\Delta p_{0}=0$ of the general curve given as Fig.2 in [11. Here however, we were also able to calculate its analytic form.

The formal position eigenstates are generally no longer orthogonal. From (23) we can now however directly read off the one-parameter family of diagonalisations of $\mathbf{x}$. The sets of eigenvectors parametrised by $\lambda \in[-1,1[$

$$
\left\{\left|\psi_{(2 n+\lambda) \hbar \sqrt{\beta}}\right\rangle \mid \quad n \in \mathbb{Z}\right\}
$$

consist of mutually orthogonal eigenvectors, since

$$
\left\langle\psi_{(2 n+\lambda) \hbar \sqrt{\beta}} \mid \psi_{\left(2 n^{\prime}+\lambda\right) \hbar \sqrt{\beta}}\right\rangle=\delta_{n, n^{\prime}}
$$

It is not difficult to see that each set is also complete. Each of these lattices of formal $\mathbf{x}$-eigenvectors has the lattice spacing $2 \hbar \sqrt{\beta}$, which is also $2 \Delta x_{0}$.

Thus in fact there are diagonalisations of the position operator. One might therefore be tempted to interpret this result such that we are now describing physics on lattices in position space; for example, compare with the approach in ref. [14, 15]. This is however not the case since the formal position eigenvectors $\left|\psi_{\lambda}\right\rangle$ are not physical states. This is because they are not in the domain of $\mathbf{p}$, which physically means that they have infinite uncertainty in momentum and in particular also infinite energy:

$$
\left\langle\psi_{\lambda}\left|\mathbf{p}^{2} / 2 m\right| \psi_{\lambda}\right\rangle=\text { divergent }
$$


This is in fact also the case for the position eigenvectors of ordinary quantum mechanics. However, since the uncertainty relation holds on every domain of symmetric operators $\mathbf{x}, \mathbf{x}^{2}, \mathbf{p}, \mathbf{p}^{2}$ we can in our generalized quantum mechanics conclude a much stronger statement:

Vectors $|\psi\rangle$ that have a well defined uncertainty in position $\Delta x_{|\psi\rangle}$ which is inside the 'forbidden gap'

$$
0 \leq \Delta x_{|\psi\rangle}<\Delta x_{0}
$$

can not have finite energy.

Thus, unlike in ordinary quantum mechanics the formal $\mathbf{x}$-eigenvectors with their vanishing $x$-uncertainty can now no longer be approximated by a series of physical states of finite energy where the uncertainty in positions would decrease to zero. Instead there is now a finite limit to the localizability.

\section{Recovering information on position}

Generally in quantum mechanics all information on position is encoded in the matrix elements of the position operator. Matrix elements can of course be calculated in any basis, e.g. also in the momentum eigenbasis. We now no longer have any position eigenbasis of physical states $|x\rangle$ whose matrix elements $\langle x \mid \psi\rangle$ would have the usual direct physical interpretation about positions. Nevertheless all information on position is of course still accessible. To this end let us study the states which realize the maximally allowed localization.

\subsection{Maximal localization states}

Let us explicitly calculate the states $\left|\psi_{\xi}^{m l}\right\rangle$ of maximal localization around a position $\xi$, i.e. states which have the properties

$$
\left\langle\psi_{\xi}^{m l}|\mathbf{x}| \psi_{\xi}^{m l}\right\rangle=\xi
$$

and

$$
(\Delta x)_{\left|\psi_{\xi}^{m l}\right\rangle}=\Delta x_{0}
$$

We know that $\Delta x_{0}$ is $\langle\mathbf{p}\rangle$ - dependent. Recall that the absolutely smallest uncertainty can only be reached for $\langle\mathbf{p}\rangle=0$.

Let us reconsider the (standard) derivation of the uncertainty relation. For each state in the representation of the Heisenberg algebra (actually we only need $|\psi\rangle$ to be in a domain where $\mathbf{x}, \mathbf{x}^{2}, \mathbf{p}$ and $\mathbf{p}^{2}$ are symmetric) we deduce from the positivity of the norm

$$
\|\left(\mathbf{x}-\langle\mathbf{x}\rangle+\frac{\langle[\mathbf{x}, \mathbf{p}]\rangle}{2(\Delta p)^{2}}(p-\langle p\rangle)\right)|\psi\rangle \| \geq 0
$$


that (note that $\langle[x, p]\rangle$ is imaginary)

$$
\left\langle\psi\left|(\mathbf{x}-\langle\mathbf{x}\rangle)^{2}-\left(\frac{|\langle[\mathbf{x}, \mathbf{p}]\rangle|}{2(\Delta p)^{2}}\right)^{2}(\mathbf{p}-\langle\mathbf{p}\rangle)^{2}\right| \psi\right\rangle \geq 0
$$

which immediately implies:

$$
\Delta x \Delta p \geq \frac{|\langle[\mathbf{x}, \mathbf{p}]\rangle|}{2}
$$

It is therefore clear that a state $|\psi\rangle$ will obey $\Delta x \Delta p=|\langle[\mathbf{x}, \mathbf{p}]\rangle| / 2$ i.e. it is on the boundary of the physically allowed region only if it obeys:

$$
\left(\mathbf{x}-\langle\mathbf{x}\rangle+\frac{\langle[\mathbf{x}, \mathbf{p}]\rangle}{2(\Delta p)^{2}}(\mathbf{p}-\langle\mathbf{p}\rangle)\right)|\psi\rangle=0
$$

In momentum space this takes the form of a differential equation

$$
\left(i \hbar\left(1+\beta p^{2}\right) \partial_{p}-\langle\mathbf{x}\rangle+i \hbar \frac{1+\beta(\Delta p)^{2}+\beta\langle\mathbf{p}\rangle^{2}}{2(\Delta p)^{2}}(p-\langle\mathbf{p}\rangle)\right) \psi(p)=0
$$

which can be solved to obtain:

$$
\psi(p)=N\left(1+\beta p^{2}\right)^{-\frac{1+\beta(\Delta p)^{2}+\beta\langle\mathbf{p}\rangle^{2}}{4 \beta(\Delta p)^{2}}} e^{\left(\frac{\langle\mathbf{X}\rangle}{i \hbar \sqrt{\beta}}-\frac{\left(1+\beta(\Delta p)^{2}+\beta\langle\mathbf{p}\rangle^{2}\right)\langle\mathbf{p}\rangle}{2(\Delta p)^{2} \sqrt{\beta}}\right) \tan ^{-1}(\sqrt{\beta} p)}
$$

The states of absolutely maximal localization can only be obtained for $\langle\mathbf{p}\rangle=0$, see eq. (9). We then choose the critical momentum uncertainty $\Delta p=1 / \sqrt{\beta}$ to get those states which are at that point on the curve of the uncertainty relation where the minimal position uncertainty is reached. These states are

$$
\psi_{\xi}^{m l}(p)=N\left(1+\beta p^{2}\right)^{-\frac{1}{2}} e^{-i \frac{\langle\mathbf{X}\rangle \tan ^{-1}(\sqrt{\beta} p)}{\hbar \sqrt{\beta}}}
$$

where

$$
1=N N^{*} \int_{-\infty}^{+\infty} \frac{d p}{\left(1+\beta p^{2}\right)^{2}}=N^{2} \frac{\pi}{2 \sqrt{\beta}}
$$

yields their normalization factor $N$.

Thus the momentum space wavefunctions $\psi_{\xi}^{m l}(p)$ of the states which are maximally localized (i.e. $\left.(\Delta x)_{\left|\psi_{\xi}^{m l}\right\rangle}=\Delta x_{0}\right)$ around a position $\xi$ (i.e. $\left\langle\psi_{\xi}^{m l}|\mathbf{x}| \psi_{\xi}^{m l}\right\rangle=\xi$ ) read:

$$
\psi_{\xi}^{m l}(p)=\sqrt{\frac{2 \sqrt{\beta}}{\pi}}\left(1+\beta p^{2}\right)^{-\frac{1}{2}} e^{-i \frac{\xi \tan ^{-1}(\sqrt{\beta} p)}{\hbar \sqrt{\beta}}}
$$

These states generalize the plane waves in momentum space or Dirac $\delta$ - 'functions' in position space which would describe maximal localization in ordinary quantum 
mechanics. Unlike the latter, the new maximal localization states are now proper physical states of finite energy

$$
\left\langle\psi_{\xi}^{m l}\left|\frac{\mathbf{p}^{2}}{2 m}\right| \psi_{\xi}^{m l}\right\rangle=\frac{2 \sqrt{\beta}}{\pi} \int_{-\infty}^{+\infty} \frac{d p}{\left(1+\beta p^{2}\right)^{2}} \frac{p^{2}}{2 m}=\frac{1}{2 m \beta} .
$$

Due to the 'fuzziness' of space the maximal localization states are in general no longer mutually orthogonal:

$$
\begin{aligned}
\left\langle\psi_{\xi^{\prime}}^{m l} \mid \psi_{\xi}^{m l}\right\rangle & =\frac{2 \sqrt{\beta}}{\pi} \int_{-\infty}^{+\infty} \frac{d p}{\left(1+\beta p^{2}\right)^{2}} e^{-i \frac{\left(\xi-\xi^{\prime}\right) \tan ^{-1}(\sqrt{\beta} p)}{\hbar \sqrt{\beta}}} \\
& =\frac{2 \sqrt{\beta}}{\pi} \int_{-\pi / 2}^{+\pi / 2} \frac{d \tilde{p}}{\sqrt{\beta}} \frac{1}{1+\frac{\sin ^{2}(\tilde{p})}{\cos ^{2}(\tilde{p})}} e^{-i \frac{\xi-\xi^{\prime}}{\hbar \sqrt{\beta}} \tilde{p}} \\
& =\frac{1}{\pi}\left(\frac{\xi-\xi^{\prime}}{2 \hbar \sqrt{\beta}}-\left(\frac{\xi-\xi^{\prime}}{2 \hbar \sqrt{\beta}}\right)^{3}\right)^{-1} \sin \left(\frac{\xi-\xi^{\prime}}{2 \hbar \sqrt{\beta}} \pi\right)
\end{aligned}
$$

The poles of the first factor are cancelled by zeros of the sine function.

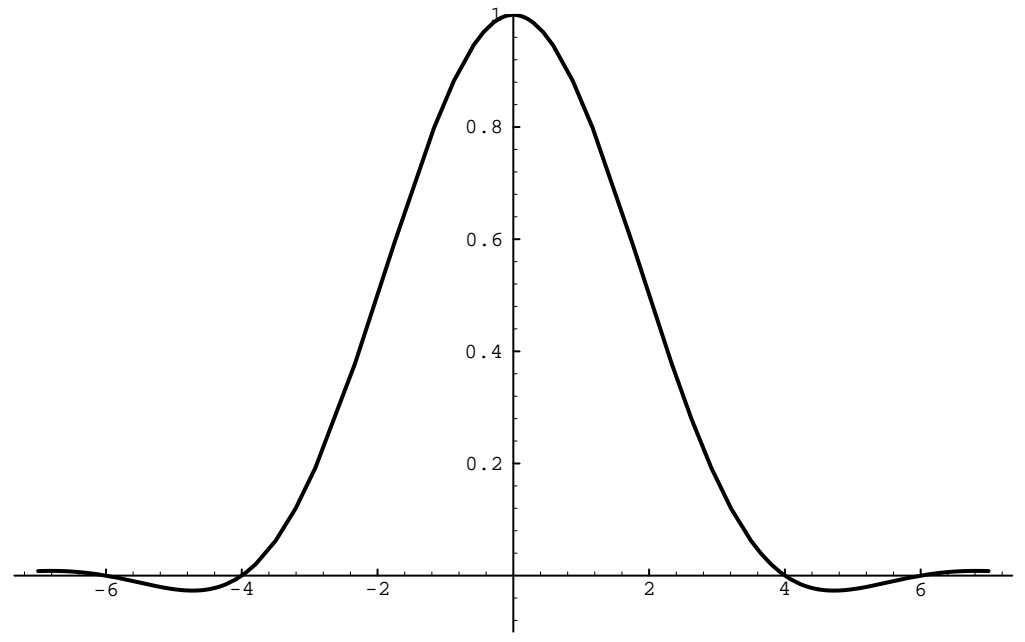

Fig3: Plotting $\left\langle\psi_{\xi^{\prime}}^{m l} \mid \psi_{\xi}^{m l}\right\rangle$ over $\xi-\xi^{\prime}$ in units of $\hbar \sqrt{\beta}=\Delta x_{0}$

For the width of the main peak note that this curve yields the overlap of two maximal localization states, each having a standard deviation $\Delta x_{0}$.

\subsection{Transformation to quasi-position wave functions}

While in ordinary quantum mechanics it is often useful to expand the states $|\psi\rangle$ in the position 'eigenbasis' $\{|x\rangle\}$ as $\langle x \mid \psi\rangle$, there are now no physical states which would 
form a position eigenbasis. Although there is a one parameter family of $\mathbf{x}$-eigenbases, due to the minimal uncertainty gap neither of these bases consists of physical states; furthermore they could not even be approximated by physical states of increasing localization.

However we can still project arbitrary states $|\phi\rangle$ on maximally localized states $\left|\psi_{\xi}^{m l}\right\rangle$ to obtain the probability amplitude for the particle being maximally localized around the position $\xi$.

Let us call the collection of these projections $\left\langle\psi_{\xi}^{m l} \mid \phi\right\rangle$ the state's 'quasi-position wavefunction' $\phi(\xi)$ :

$$
\phi(\xi):=\left\langle\psi_{\xi}^{m l} \mid \phi\right\rangle
$$

In the limit $\beta \rightarrow 0$ the ordinary position wave function $\phi(\xi)=\langle\xi \mid \phi\rangle$ is recovered.

The transformation of a state's wavefunction in the momentum representation into its quasiposition wave function is of course:

$$
\psi(\xi)=\sqrt{\frac{2 \sqrt{\beta}}{\pi}} \int_{-\infty}^{+\infty} \frac{d p}{\left(1+\beta p^{2}\right)^{3 / 2}} e^{\frac{i \xi \tan ^{-1}(\sqrt{\beta} p)}{\hbar \sqrt{\beta}}} \psi(p)
$$

The quasi-position wavefunction of a momentum eigenstate $\psi_{\tilde{p}}(p)=\delta(p-\tilde{p})$, of energy $E=\tilde{p}^{2} / 2 m$, is still a plane wave. However, for its wavelength we obtain a modified dispersion relation:

$$
\lambda(E)=\frac{2 \pi \hbar \sqrt{\beta}}{\tan ^{-1}(\sqrt{2 m \beta E})}
$$

The existence of a limit to the precision to which positions can be resolved manifests itself in the fact that, since the $\tan ^{-1}$ is bounded, there is a nonzero minimal wavelength. The Fourier decomposition of the quasi-position wave function of physical states does not contain wavelength components smaller than

$$
\lambda_{0}=4 \hbar \sqrt{\beta} .
$$

Note that, in contrast to ordinary quantum mechanics, since

$$
E(\lambda)=\left(\tan \frac{2 \pi \hbar \sqrt{\beta}}{\lambda}\right)^{2} \frac{1}{2 m \beta}
$$

quasi-position wavefunctions can no longer have arbitrarily fine 'ripples', since the energy of the short wavelength modes diverges as the wavelength approaches the finite value $\lambda_{0}$.

The transformation (43) that maps momentum space wave functions into quasiposition space wave functions is the generalization of the Fourier transformation and is still invertible. Explicitly, the transformation of a quasi-position wavefunction into a momentum space wave function is easily checked to be:

$$
\psi(p)=\frac{1}{\sqrt{8 \pi \sqrt{\beta}} \hbar} \int_{-\infty}^{+\infty} d \xi\left(1+\beta p^{2}\right)^{1 / 2} e^{-i \xi \frac{\tan ^{-1}(\sqrt{\beta} p)}{\hbar \sqrt{\beta}}} \psi(\xi)
$$


Compare also with the generalized Fourier transformation of the discretized quantum mechanics in [15, 16].

\subsection{Quasi-position representation}

The Heisenberg algebra has a representation on the space of quasi-position wave functions which we now describe. Using eqs. (14) and (43) the scalar product of states in terms of the quasi-position wavefunctions can be written as

$$
\begin{aligned}
\langle\psi \mid \phi\rangle & =\int_{-\infty}^{+\infty} \frac{d p}{1+\beta p^{2}} \psi^{*}(p) \phi(p) \\
& =\left(8 \pi \hbar^{2} \sqrt{\beta}\right)^{(-1)} \int_{-\infty}^{+\infty} \int_{-\infty}^{+\infty} \int_{-\infty}^{+\infty} d p d \xi d \xi^{\prime} e^{i\left(\xi-\xi^{\prime}\right) \frac{\tan ^{-1}(\sqrt{\beta} p)}{\hbar \sqrt{\beta}}} \psi^{*}(\xi) \phi\left(\xi^{\prime}\right)
\end{aligned}
$$

We see from (47) that the momentum operator is represented as

$$
\mathbf{p} . \psi(\xi)=\frac{\tan \left(-i \hbar \sqrt{\beta} \partial_{\xi}\right)}{\sqrt{\beta}} \psi(\xi)
$$

on the quasi-position wave functions. From the action of $\mathbf{x}$ on momentum space wave functions and eq. (47) we derive its action on the quasi-position wave functions:

$$
\mathbf{x} . \psi(\xi)=\left(\xi+\beta \frac{\tan \left(-i \hbar \sqrt{\beta} \partial_{\xi}\right)}{\sqrt{\beta}}\right) \psi(\xi)
$$

We pause to comment on some important features of the quasi-position representation. We found that the position and momentum operators $\mathbf{x}, \mathbf{p}$ can be expressed in terms of the multiplication and differentiation operators $\xi,-i \hbar \partial_{\xi}$ which obey the commutation relations of ordinary quantum mechanics. However, this does not mean that we are still dealing with the same space of physical states with the same properties as in ordinary quantum mechanics. The scalar product (49) of quasi-position wave functions reduces to the ordinary scalar product on position space only for $\beta \rightarrow 0$. Recall also that the quasi-position wave functions of physical states Fourier decompose into wavelengths strictly larger than a finite minimal wavelength. It is only on such physical wave functions that the momentum operator is defined. On general functions of $\xi$ the power series in the $-i \hbar \partial_{\xi}$ which forms the tan would not be convergent. In addition the position operator is not diagonalizable in any domain of the symmetric operators $\mathbf{x}^{2}$ and $\mathbf{p}^{2}$; in particular the quasi-position representation does not diagonalise it. The main advantage of the quasi-position representation is that it has a direct physical interpretation. Recall that $\psi(\xi)$ is the probability amplitude for finding the particle maximally localized around the position $\xi$, i.e. with standard deviation $\Delta x_{0}$.

Let us close with some general remarks on the existence of transformations to ordinary quantum mechanics and on the significance of the fact that those transformations are noncanonical. 
There are (in $n \geq 1$ dimensions) algebra homomorphisms from generalised Heisenberg algebras $\mathcal{H}$ generated by operators $\mathbf{x}$ and $\mathbf{p}$ to the ordinary Heisenberg algebra $\mathcal{H}_{0}$ generated by operators $\mathbf{x}_{0}$ and $\mathbf{p}_{0}$. In one dimension we have e.g. the algebra homomorphism $h: \mathcal{H} \rightarrow \mathcal{H}_{0}$ which acts on the generators as: $h: \mathbf{p} \rightarrow \mathbf{p}_{0}$, $h: \mathbf{x} \rightarrow \mathbf{x}_{0}+\beta \mathbf{p}_{0} \mathbf{x}_{0} \mathbf{p}_{0}$. Such mappings $h$ are of representation theoretic interest since they induce to any representation $\rho$ of $\mathcal{H}_{0}$ a representation $\rho_{h}:=\rho \circ h$ of the new Heisenberg algebra $\mathcal{H}$.

Crucially however, all $h$ are noncanonical. In fact, since unitary transformations generally preserve the commutation relations no representation of $\mathcal{H}$ is unitarily equivalent to any representation of $\mathcal{H}_{0}$. Therefore the set of predictions, such as expectation values or transition amplitudes, of a system based on the new position and momentum operators cannot be matched by the set of predictions of any system that is based on position and momentum operators obeying the ordinary commutation relations.

\section{A relevant example: The harmonic oscillator}

We would like now to apply the formalism developed thus far to the interesting case of a linear harmonic oscillator, deducing both the energy spectrum and the expression of the corresponding eigenfunctions. The comparison with the limiting case $\beta=0$ will be particularly interesting.

From the expression for the Hamiltonian:

$$
H=\frac{\mathbf{p}^{2}}{2 m}+m \omega^{2} \frac{\mathbf{x}^{2}}{2}
$$

and the representation for $\mathbf{x}$ and $\mathbf{p}$ in the $\mathbf{p}$-space reported in Section 3.2 we get the following form for the stationary state Schrödinger equation:

$$
\frac{d^{2} \psi(p)}{d p^{2}}+\frac{2 \beta p}{1+\beta p^{2}} \frac{d \psi(p)}{d p}+\frac{1}{\left(1+\beta p^{2}\right)^{2}}\left[\epsilon-\eta^{2} p^{2}\right] \psi(p)=0
$$

where we have defined:

$$
\epsilon=\frac{2 E}{m \hbar^{2} \omega^{2}} ; \quad \eta^{2}=\frac{1}{(m \hbar \omega)^{2}}
$$

and $E$ is the energy.

The usual Schrödinger equation $(\beta=0)$ for the linear harmonic oscillator only has one singularity at infinity, which is not, however, of the Fuchsian kind [18]. In that case the well-known procedure is to write the solution as the product of a decreasing gaussian factor and of a new function satisfying an equation, leading to Hermite polynomials, where the quadratic term $\eta^{2} p^{2}$ is cancelled in the differential equation if the gaussian factor is properly chosen. From eq. (52) we see that the introduction 
of a finite value for $\beta$ completely changes the singularity structure in the complex plane. Three singular points are now present: the usual point at infinity as well as $p= \pm i / \sqrt{\beta}$. These are all regular since the coefficient of the first derivative term only behaves as a simple pole in the neighbourhood of each singularity; the one in front of the function itself contains only double polest. Qualitatively the presence of a minimal length softens the behaviour of the wave equation at very large momenta, transforming the point at infinity into a Fuchsian singularity.

Equation (52) is a Riemann equation whose solution is given in terms of hypergeometric functions, which can always be expressed in terms of the Gauss hypergeometric series, up to some simple factors. In order to find the explicit solution it is useful to introduce, as usual, a new variable $\zeta$ in terms of which the poles are shifted to the reference values 0,1 and $\infty$ :

$$
\zeta=\frac{1}{2}+i \frac{\sqrt{\beta}}{2} p
$$

Equation (52) then reads:

$$
\frac{d^{2} \psi(\zeta)}{d \zeta^{2}}+\frac{2 \zeta-1}{\zeta(\zeta-1)} \frac{d \psi(\zeta)}{d \zeta}-\frac{q+r(1-2 \zeta)^{2}}{\zeta^{2}(\zeta-1)^{2}} \psi(\zeta)=0
$$

with:

$$
q=\epsilon / 4 \beta ; \quad r=\eta^{2} / 4 \beta^{2}
$$

whose solution is represented by the Riemann symbol:

$$
P\left(\begin{array}{cccc}
0 & 1 & \infty & \\
\alpha_{1} & \beta_{1} & \gamma_{1} & ; \zeta \\
\alpha_{2} & \beta_{2} & \gamma_{2} &
\end{array}\right)
$$

From a straightforward computation one gets, for the roots $\alpha_{i}, \beta_{i}$ and $\gamma_{i}$ [18]:

$$
\begin{aligned}
& \alpha_{1}=-\sqrt{q+r} ; \quad \alpha_{2}=\sqrt{q+r} \\
& \beta_{1}=-\sqrt{q+r} ; \quad \beta_{2}=\sqrt{q+r} \\
& \gamma_{1}=\frac{1}{2}(1-\sqrt{1+16 r}) ; \quad \gamma_{2}=\frac{1}{2}(1+\sqrt{1+16 r})
\end{aligned}
$$

The solution (57) is simply related to the solution of the hypergeometric Gauss equation $F(a, b ; c ; z)$, for which one of the roots for both the singularities at finite distance is zero

$$
\begin{aligned}
P\left(\begin{array}{cccc}
0 & 1 & \infty & \\
\alpha_{1} & \beta_{1} & \gamma_{1} & ; \zeta \\
\alpha_{2} & \beta_{2} & \gamma_{2}
\end{array}\right) & =\zeta^{\alpha_{1}}(1-\zeta)^{\beta_{1}} P\left(\begin{array}{cccc}
0 & 1 & \infty \\
0 & 0 & a & ; \zeta \\
1-c & c-a-b & b
\end{array}\right) \\
& =\zeta^{\alpha_{1}}(1-\zeta)^{\beta_{1}} F(a, b ; c ; \zeta)
\end{aligned}
$$

\footnotetext{
${ }^{1}$ We recall that in order to study the singular point at infinity one should rewrite the equation in terms of the new variable $p^{\prime}=1 / p$, shifting the singularity to the origin.
} 
where

$$
\begin{aligned}
& a=\gamma_{1}-\alpha_{1}-\beta_{1}=\frac{1}{2}(1-\sqrt{1+16 r})-2 \sqrt{q+r} \\
& b=\gamma_{2}-\alpha_{1}-\beta_{1}=\frac{1}{2}(1+\sqrt{1+16 r})-2 \sqrt{q+r} \\
& c=1-2 \sqrt{q+r}
\end{aligned}
$$

We therefore finally get, in terms of the real momentum variable $p$, the general form for the solution of the equation (52):

$$
\psi(p) \propto \frac{1}{\left(1+\beta p^{2}\right)^{\sqrt{q+r}}} \cdot F\left(a, b ; c ; \frac{1}{2}+i \frac{\sqrt{\beta}}{2} p\right)
$$

Since we know that for $\beta=0$ the eigenfunctions are simply the product of a Gaussian factor with Hermite polynomials, we now look for the solutions for $\beta \neq 0$ in the cases where the hypergeometric series $F(a, b ; c, z)$ reduces to a polynomial. This is known to occur whenever $a$ or $b$ is a negative integer:

$$
\begin{array}{ll}
a=-n & \Rightarrow \sqrt{q+r}=\frac{1}{2}\left(n+\frac{1}{2}\right)-\frac{1}{4} \sqrt{1+16 r} \\
b=-n & \Rightarrow \sqrt{q+r}=\frac{1}{2}\left(n+\frac{1}{2}\right)+\frac{1}{4} \sqrt{1+16 r}
\end{array}
$$

In both cases $F(a, b ; c ; z)$ becomes a polynomial of degree $n$. However if we choose $a=-n$ the wavefunction would not have the correct behaviour at infinity and, in particular, will not belong to the domain of $\mathbf{p}^{2}$. From equation (62) one has in fact, for large $p$

$$
\psi(p) \propto \frac{1}{\left(1+\beta p^{2}\right)^{\sqrt{q+r}}} \cdot F\left(-n, b ; c ; \frac{1}{2}+i \frac{\sqrt{\beta}}{2} p\right) \sim p^{(\sqrt{1+16 r}-1) / 2}
$$

which diverges. Hence the condition $b=-n$ yields the energy spectrum and the corresponding proper eigenfunctions. In this case $\sqrt{q+r}>0$ for any $n$ and for large $p$ the wavefunction behaves as

$$
\psi(p) \propto \frac{1}{\left(1+\beta p^{2}\right)^{\sqrt{q+r}}} \cdot F\left(a,-n ; c ; \frac{1}{2}+i \frac{\sqrt{\beta}}{2} p\right) \sim p^{-(\sqrt{1+16 r}+1) / 2}
$$

and so is normalizable with respect to the measure $d p /\left(1+\beta p^{2}\right)$. It also belongs to the domain of $\mathbf{p}^{2}$, as it is immediately checked. Note that, for any fixed $n$, the larger the value of $r$ (i.e. the smaller the value of $\beta$ ), the more rapid the decay to zero of the wavefunction at infinity. In particular in the limit $\beta \rightarrow 0$, using (63), we recover the usual gaussian behaviour of the harmonic oscillator wavefunctions

$$
\lim _{\beta \rightarrow 0} \frac{1}{\left(1+\beta p^{2}\right)^{\sqrt{q+r}}}=\exp \left(-\frac{\eta^{2} p^{2}}{2}\right)
$$


Hence to each quantum number $n$ there corresponds the eigenfunction

$$
\psi_{n}(p) \propto \frac{1}{\left(1+\beta p^{2}\right)^{\sqrt{q+r}_{n}}} \cdot F\left(a_{n},-n ; c_{n} ; \frac{1}{2}+i \frac{\sqrt{\beta}}{2} p\right)
$$

where

$$
\sqrt{q+r}_{n}=\frac{1}{2}\left(n+\frac{1}{2}\right)+\frac{1}{4} \sqrt{1+16 r} ; a_{n}=-n-\sqrt{1+16 r} ; c_{n}=1-2 \sqrt{q+r_{n}}
$$

For the energy spectrum we obtain from (63) and (56):

$$
E_{n}=\hbar \omega\left(n+\frac{1}{2}\right)\left(\frac{1}{4 \sqrt{r}}+\sqrt{1+\frac{1}{16 r}}\right)+\hbar \omega \frac{1}{4 \sqrt{r}} n^{2}
$$

Notice that the usual spectrum is recovered in the limit $\beta \rightarrow 0$ (or $r \rightarrow \infty$ ); for finite $\beta$, the energy levels also depend on the square of the quantum number $n$, and asymptotically, for large $n$, they grow as $n^{2}$. In Figure 4 we illustrate for comparison the values of the ratio $E_{n} / \hbar \omega$ for the usual harmonic oscillator and for $r=100$.

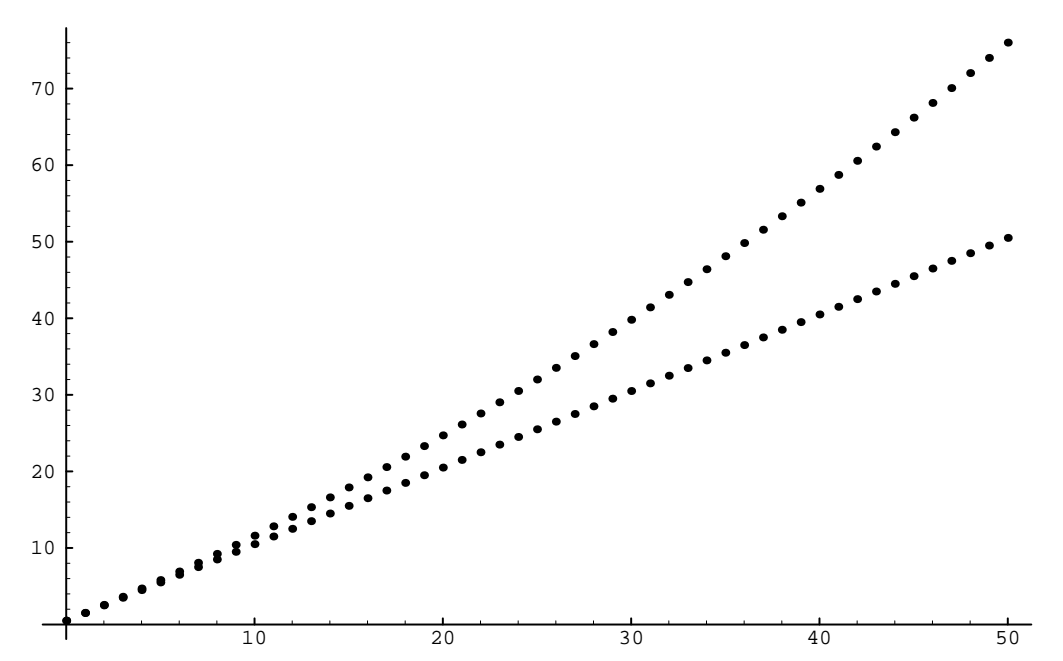

Fig4: Comparing $E_{n} / \hbar \omega$ for $r=100$ with the harmonic oscillator spectrum of ordinary quantum mechanics

We do here not prove the completeness of the set of eigenfunctions $\left\{\psi_{n}(p)\right\}$, which is quite obvious since the $\psi_{n}(p)$ adiabatically reduce, in the limit $\beta=0$, to the ordinary harmonic oscillator eigenfunctions whose completeness is known.

\section{Generalisation to $n$ dimensions}

We now turn to extending the formalism developed in the previous sections to $n$ spatial dimensions. Our aim is to study an $n$-dimensional generalization of the framework 
which still allows the use of our powerful momentum space representation.

\subsection{Generalised Heisenberg algebra for $n$ dimensions}

A natural generalization of (6) which preserves the rotational symmetry is:

$$
\left[\mathbf{x}_{i}, \mathbf{p}_{j}\right]=i \hbar \delta_{i j}\left(1+\beta \overrightarrow{\mathbf{p}}^{2}\right)
$$

It implies nonzero minimal uncertainties in each position coordinate.

A more general situation including nonzero minimal uncertainties in momenta has been studied in 11]. For the construction of Hilbert space representations for this general case one cannot work on position space and one has to resort e.g. to a generalized Bargmann Fock representation. Here we will specialize to the situation with nonzero minimal uncertainties in positions only.

We require

$$
\left[\mathbf{p}_{i}, \mathbf{p}_{j}\right]=0
$$

which allows us to straightforwardly generalize the momentum space representation of the previous sections to $n$ dimensions:

$$
\begin{aligned}
& \mathbf{p}_{i} \cdot \psi(p)=p_{i} \psi(p) \\
& \mathbf{x}_{i} \cdot \psi(p)=i \hbar\left(1+\beta \vec{p}^{2}\right) \partial_{p_{i}} \psi(p)
\end{aligned}
$$

This fixes the commutation relations among the position operators. Explicitly we have

$$
\left[\mathbf{x}_{i}, \mathbf{x}_{j}\right]=2 i \hbar \beta\left(\mathbf{p}_{i} \mathbf{x}_{j}-\mathbf{p}_{j} \mathbf{x}_{i}\right)
$$

leading naturally to a 'noncommutative geometric' generalization of position space. Compare also with the approach [19].

Note that the generalization to the case

$$
\left[\mathbf{x}_{i}, \mathbf{p}_{j}\right]=i \hbar \delta_{i j}\left(1+f\left(\overrightarrow{\mathbf{p}}^{2}\right)\right)
$$

is straightforward, yielding

$$
\mathbf{x}_{i} \cdot \psi(p)=i \hbar\left(1+f\left(\overrightarrow{\mathbf{p}}^{2}\right)\right) \frac{\partial}{\partial p_{i}} \psi(p)
$$

and

$$
\left[\mathbf{x}_{i}, \mathbf{x}_{j}\right]=-2 i \hbar f^{\prime}\left(\overrightarrow{\mathbf{p}}^{2}\right)\left(\mathbf{x}_{i} \mathbf{p}_{j}-\mathbf{x}_{j} \mathbf{p}_{i}\right)
$$

Here we restrict ourselves to the case $f\left(\overrightarrow{\mathbf{p}}^{2}\right)=\beta \overrightarrow{\mathbf{p}}^{2}$.

The operators $\mathbf{x}_{i}$ and $\mathbf{p}_{j}$ are symmetric on the domain $S_{\infty}$ with respect to the scalar product:

$$
\langle\psi \mid \phi\rangle=\int_{-\infty}^{+\infty} \frac{d^{n} p}{1+\beta \vec{p}^{2}} \psi^{*}(p) \phi(p)
$$


The identity operator can be expanded as

$$
1=\int_{-\infty}^{+\infty} \frac{d^{n} p}{1+\beta \vec{p}^{2}}|p\rangle\langle p|
$$

and the scalar product of momentum eigenstates is therefore:

$$
\left\langle p \mid p^{\prime}\right\rangle=\left(1+\beta \vec{p}^{2}\right) \delta^{n}\left(p-p^{\prime}\right)
$$

While the momentum operators are still essentially self-adjoint, the position operators are merely symmetric and do not have physical eigenstates. Maximal localization states can again be used to define quasi-position wave functions. We shall omit a detailed discussion of the functional analysis which is completely analogous to the one-dimensional situation. The quasi-position analysis will however be somewhat more involved. We now focus on the action of the rotation group.

\subsection{Representation of the rotation group}

The commutation relations $(70,71,74)$ do not break the rotational symmetry. In fact, the generators of rotations can still be expressed in terms of the position and momentum operators as

$$
\mathbf{L}_{i j}:=\frac{1}{1+\beta \overrightarrow{\mathbf{p}}^{2}}\left(\mathbf{x}_{i} \mathbf{p}_{j}-\mathbf{x}_{j} \mathbf{p}_{i}\right)
$$

which in 3 dimensions can be written as

$$
\mathbf{L}_{k}:=\frac{1}{1+\beta \overrightarrow{\mathbf{p}}^{2}} \epsilon_{i j k} \mathbf{x}_{i} \mathbf{p}_{j}
$$

generalizing the usual definition of orbital angular momentum. Note that $1 /\left(1+\beta \overrightarrow{\mathbf{p}}^{2}\right)$ is an unproblematic bounded operator acting on the momentum space wave functions as multiplication by $1 /\left(1+\beta \vec{p}^{2}\right)$. The representation of the generators of rotations on momentum wave functions is

$$
\mathbf{L}_{i j} \psi(p)=-i \hbar\left(p_{i} \partial_{p_{j}}-p_{j} \partial_{p_{i}}\right) \psi(p)
$$

where

$$
\begin{gathered}
{\left[\mathbf{p}_{i}, \mathbf{L}_{j k}\right]=i \hbar\left(\delta_{i k} \mathbf{p}_{j}-\delta_{i j} \mathbf{p}_{k}\right)} \\
{\left[\mathbf{x}_{i}, \mathbf{L}_{j k}\right]=i \hbar\left(\delta_{i k} \mathbf{x}_{j}-\delta_{i j} \mathbf{x}_{k}\right)} \\
{\left[\mathbf{L}_{i j}, \mathbf{L}_{k l}\right]=i \hbar\left(\delta_{i k} \mathbf{L}_{j l}-\delta_{i l} \mathbf{L}_{j k}+\delta_{j l} \mathbf{L}_{i k}-\delta_{j k} \mathbf{L}_{i l}\right)}
\end{gathered}
$$

equivalent to ordinary quantum mechanics. However we also have

$$
\left[\mathbf{x}_{i}, \mathbf{x}_{j}\right]=-2 i \hbar \beta\left(1+\beta \overrightarrow{\mathbf{p}}^{2}\right) \mathbf{L}_{i j}
$$


showing that we have a noncommutative geometry. We therefore have the uncertainty relations

$$
\Delta x_{i} \Delta p_{j} \geq \frac{\hbar}{2} \delta_{i j}\left(1+\beta \sum_{k=1}^{n}\left(\left(\Delta p_{k}\right)^{2}+\left\langle\mathbf{p}_{k}\right\rangle^{2}\right)\right)
$$

yielding the minimal uncertainties $\Delta x_{0}=\hbar \sqrt{\beta}$ for each coordinate, and

$$
\Delta x_{i} \Delta x_{j} \geq \beta \hbar\left\langle\left(1+\beta \overrightarrow{\mathbf{p}}^{2}\right) \mathbf{L}_{i j}\right\rangle
$$

It is straightforward to check that the algebra generated by (84 87) respects the Jacobi identities.

E.g. in three dimensions one easily verifies that we now have the commutation relations:

$$
\begin{gathered}
{\left[\mathbf{p}_{i}, \mathbf{L}_{j}\right]=i \hbar \epsilon_{i j k} \mathbf{p}_{k}} \\
{\left[\mathbf{x}_{i}, \mathbf{L}_{j}\right]=i \hbar \epsilon_{i j k} \mathbf{x}_{k}} \\
{\left[\mathbf{L}_{i}, \mathbf{L}_{j}\right]=i \hbar \epsilon_{i j k} \mathbf{L}_{k}} \\
{\left[\mathbf{x}_{i}, \mathbf{x}_{j}\right]=-2 i \hbar \beta\left(1+\beta \overrightarrow{\mathbf{p}}^{2}\right) \epsilon_{i j k} \mathbf{L}_{k}}
\end{gathered}
$$

where

$$
\mathbf{L}_{k} . \psi(p)=-i \hbar \epsilon_{i j k} p_{i} \partial_{p_{j}} \psi(p)
$$

is the action of the angular momentum operator on wavefunctions.

\section{Outlook}

The implications of the introduction of a nonzero minimal length in quantum mechanics are quite profound. We have shown in the simplest non-trivial case that it is no longer possible to spatially localize a wavefunction to arbitrary precision. The best one can do is consider the set of maximally localized states as discussed in section IV. These states have a modified deBroglie relation (44), and cannot contain wavelength components smaller than the minimal value $\lambda_{0}$ given by (45). The harmonic oscillator example in section $\mathrm{V}$ shows that the energy levels of a given system can deviate significantly from the usual quantum mechanical case once energy scales become comparable to the scale $\sqrt{\beta}$. Although the onset of this scale is an empirical question, it is presumably set by quantum gravitational effects.

We have three possible fields of application in mind to extend our work further. First of all, and most fascinatingly, a minimal length of the form of a minimal position uncertainty may indeed describe a genuine feature of spacetime, arising directly from gravity. The new kind of short distance regularisation therefore has to be implemented into quantum field theory. One possibility is to take the general approach 12] of employing the path integral framework, in which the action functional $S[\phi]$ can generally be expressed in terms of the scalar product $s p($,$) and pointwise multiplica-$ tion $*$ of fields, where $x_{\mu}$ and $p^{\nu}$ are formal operators that act on the fields. Given a 
Lagrangian the strategy is to stick to the basis invariant form of its action functional, while generalising the commutation relations of the $x_{\mu}$ and $p^{\nu}$. The modified representation theory then fixes the action of the operators, the form of the scalar product and also largely (though not completely) determines the generalisation of the pointwOAise multiplication of fields i.e. the generalisation of local interactions. This then leads to generalised Feynman rules. The general studies in [12] have been carried out in a generalised Bargmann Fock representation, which is somewhat difficult to handle. The techniques that we have obtained here, in particular the quasiposition representation, should now allow a much more detailed examination of the phenomenon of a minimal position uncertainty in quantum field theory than has been possible so far. For example interaction terms, which would be slightly nonlocal on conventional spacetime, may now be strictly local in the sense that (to the extent that short distances can be resolved) no nonlocality could be observed because of the intrinsic minimal position uncertainties. A forthcoming paper on this is in progress.

Related to these studies is of course a second possible field of application, namely the development of a new regularisation method which does not change the spacetime dimension.

Finally, a quantum theory with minimal uncertainties might be useful as an effective theory of nonpointlike particles. These could, for example, be strings since our formalism includes the uncertainty relation obtained in string theory, see e.g. [7]. In addition, this suggests investigating whether our formalism may also be used to effectively describe compound particles such as mesons in situations where their nonzero size matters but where details of their internal structure does not contribute. The parameter $\beta$ would then of course not be at the Planck scale but at a scale that would relate the 'extension' of the particle to the minimal uncertainty. Furthermore, since quasi-particles and collective excitations cannot be localised to arbitrary precision, there might be a possibility of including some of the first order dynamical behavior of such systems in the kinematical effects of generalised uncertainty relations, thereby improving (or at least simplifying) the effective description.

\section{References}

[1] N.N. Khuri, hep-ph/9405406, Preprint RU-94-5-B (1994).

[2] P. K. Townsend, Phys. Rev. D15, 2795 (1976).

[3] M-T. Jaeckel, S. Reynaud, Phys. Lett. A185, 143 (1994)

[4] D. Amati, M. Cialfaloni, G. Veneziano, Phys. Lett. B216, 41 (1989).

[5] A.P. Balachandran, G. Bimonte, E. Ercolessi, G. Landi, F. Lizzi, G. Sparano, P. Teotonio-Sobrinho, Preprint ICTP: IC/94/38, DSF-T-2/94, SU-4240-567 (1994). 
[6] K. Konishi, G. Paffuti, P. Provero, Phys. Lett. B234, 276 (1990).

[7] M. Maggiore, Phys. Lett. B319, 83 (1993); M.Maggiore, Phys.Rev. D49, 5182 (1994).

[8] L. J. Garay, Int. J. Mod. Phys. A10 145-166 (1995).

[9] A. Kempf, Lett. Math. Phys. 26, 1 (1992).

[10] A. Kempf, J. Math. Phys.34, 969 (1993).

[11] A. Kempf, J. Math. Phys. 35, 4483 (1994).

[12] A. Kempf, Preprint DAMTP/94-33, hep-th/9405067 (1994).

[13] L. D. Faddeev, N. Yu. Reshetikhin, L. A. Takhtajan, Alg. Anal. 1, 178 (1989).

[14] J. Schwenck, J. Wess, Phys. Lett. B291, 273 (1992).

[15] A. Hebecker, S. Schreckenberg, J. Schwenk, W. Weich, J. Wess, Lett. Math. Phys. 31, 279 (1994).

[16] T.H. Koornwinder, R.F. Swarttouw, Trans. AMS 333, 445 (1992).

[17] S. Majid, Class. Quantum Grav. 5, 1587 (1988).

[18] V. I. Smirnov, A course of Higher Mathematics, Vol. III, Pergamon Press (1964).

[19] S. Doplicher, K. Fredenhagen, J. E. Roberts, Phys. Lett.B331, 39 (1994). 